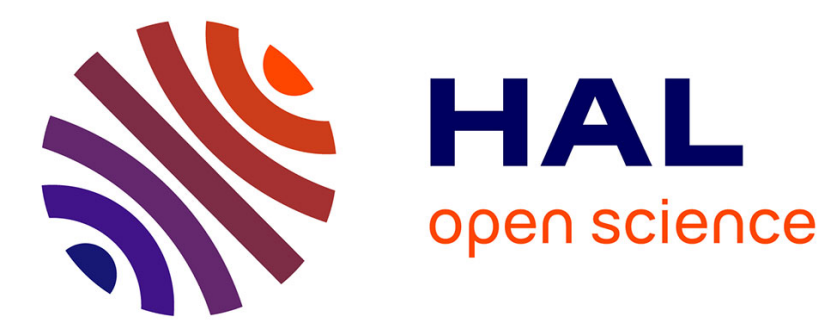

\title{
Experimental implementation of an Ultra-Wide Band MIMO radar
}

Ettien Lazare Kpré, Thomas Fromenteze, Cyril Decroze, David Carsenat

\section{To cite this version:}

Ettien Lazare Kpré, Thomas Fromenteze, Cyril Decroze, David Carsenat. Experimental implementation of an Ultra-Wide Band MIMO radar. Eumw-Eurad 2015, Sep 2015, Paris, France. hal-01212081

\section{HAL Id: hal-01212081 \\ https://hal.science/hal-01212081}

Submitted on 20 Oct 2015

HAL is a multi-disciplinary open access archive for the deposit and dissemination of scientific research documents, whether they are published or not. The documents may come from teaching and research institutions in France or abroad, or from public or private research centers.
L'archive ouverte pluridisciplinaire HAL, est destinée au dépôt et à la diffusion de documents scientifiques de niveau recherche, publiés ou non, émanant des établissements d'enseignement et de recherche français ou étrangers, des laboratoires publics ou privés. 


\title{
Experimental implementation of an Ultra-Wide Band MIMO radar
}

\author{
Ettien L. Kpré, Thomas Fromenteze, Cyril Decroze, David Carsenat, \\ Xlim Research Institute, Limoges University, France \\ Email : ettien-lazare.kpre@unilim.fr
}

\begin{abstract}
In this paper, a radar system implementation is addressed to detect a point target, that is a Multiple-Input Multiple-Output (MIMO) Radar. It is an emergent concept in radar field and has reached a substantial considerations. The advantages of MIMO radars is presented in comparison of SIMO and SAR radars. The simulation and measurement results show that the MIMO Radars can reach a better angular resolution while keeping a small number of antennas.
\end{abstract}

\section{INTRODUCTION}

Microwave imaging has been an important field of research and development, thanks to its performances in the terms of military and civilian applications. MIMO radars have received great interest over recent years [2]-[3] due to their benefits compared to conventional radars. The essence of this concept is to probe the channel with $\mathrm{M}$ orthogonal signals and record the backscattered signals with $\mathrm{N}$ receivers which are spatially independents to the transmitters. Thus the received signal from each transmitter can be separated from others by correlation in pairs. The scattered signal for each couple transmitter/receiver provides two main benefits : spatial diversity gain [1] and increased degree-of-freedoms (DOFs)[4]. Each couple provides an information about the probed channel as shown in Fig.1.

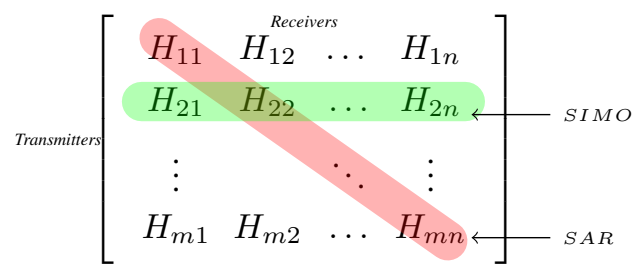

Figure 1: Illustration of a Radar MIMO scattering matrix. $H_{i j}$ denotes the scattered signal from the $i^{t h}$ transmitter to the $j^{t h}$ receiver.

According to this general definition, many traditional radars can be considered as a special case of MIMO radar. The synthetic Aperture radar which consists on moving antennas to create a synthetic aperture will measure informations corresponding to the diagonal of the channel matrix $H$, whereas the Single-Input Multiple-Output radar is similar to the measurement of one row of the matrix $H$. The remainder of the paper is organized as follows: MIMO scattering matrix formulation is first considered in the Section II focusing on the benefits of MIMO radars. Simulation and experimental results are then presented in Section III \& IV to validate the concept.

\section{MIMO SCATTERING MATRIX FORMULATION}

The term MIMO means any radar system that probes a channel by transmitting independent waveforms and received with some specific signal processing. For a one-shot measurement of the matrix elements, waveforms should the orthogonal in order to separate each couple of transmitter/receiver. Thus, waveform design is significant issue associated with MIMO Radar [5], but it is not addressed in this paper. For every examples discussed here, signal separation has been exploited to enable the scattering matrix elements $H_{i j}$.

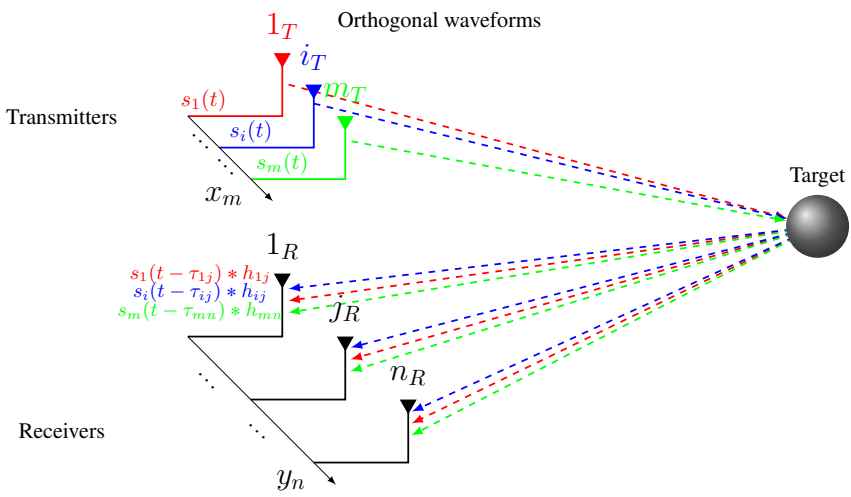

Figure 2: Illustration of a Radar MIMO principle. $h_{i j}$ represents the MIMO matrix components due to $i^{t h}$ transmitter and the $j^{\text {th }}$ receiver. $x_{m}$ and $y_{n}$ are respectively the transmitters and the receivers positions.

The received signal from $n T$ transmitters can be expressed in frequency domain as :

$$
S_{R}=\sum_{i=0}^{n T-1}[H] . S_{i}+N_{G}
$$

where $N_{G}$ is the additive Gaussian noise, $S$ the Fourier transform of transmitted signals, $H$ the scattering matrix that contains the probed channel informations and expressed as :

$$
(H)_{m, n} \propto \exp \left(-j k \cdot\left(x_{m}+y_{n}\right) \cdot d \cdot \sin \left(\theta_{0}\right)\right)
$$

where $\theta_{0}$ refers to the signal Direction of Arrival (DoA) while considering the target at far-field distance from the radar system and $k$ the wavenumber. This channel matrix contains informations referring to the target parameters. After estimation of the channel matrix, a classic beamforming in time domain algorithm is performed to detect the target position. 


\section{Simulation RESUlts}

Let's consider a monostatic MIMO array of $M$ transmitters and $N$ receivers $(M=N=4)$ with an inter element spacing of $d=0.7 \times \lambda_{c}$. A point target with an isotropic RCS is placed at a distance $r>2 \times D^{2} / \lambda_{c}$ (far-field condition) with $\lambda_{c}$ the central wavelength in the band $3-6 G h z$. Fig. 3 shows the simulation setup.

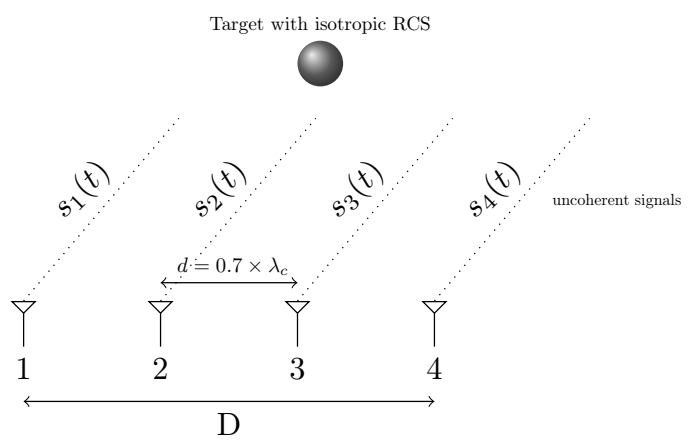

Figure 3: Computing setup. A target with an isotropic RCS placed at the far-field of a monostatic radar array of 4 elements.

When this array is used in a conventional phased radar system, each antenna transmit a full scaled waveform forming a strong transmit beam in the desired direction. Beamforming is performed only by the receivers array. Thus, the degree of freedom is $N$. However MIMO beamforming is performed by both transmitters and receivers, increasing the degree of freedom up to $M \times N$. While conventional radars employ only spatial diversity, MIMO radars employ both spatial and waveform diversity to improve spatial resolution, targets parameters identifiability and discrimination.

It has been shown in [3] that MIMO radar provides the same resolution with its equivalent phased radar while keeping a small number of antennas. This phenomenon is explained by the spatial convolution of the transmitters and receivers arrays that creates an enlarged virtual array aperture. In this example the virtual array consists of $\mathrm{N}+\mathrm{M}-1$ elements with an inter-element spacing of $d=0.7 \lambda_{c}$. Some elements are redundant as shown in Fig.4. As consequence, the design of the MIMO radar array is an important issue associated with MIMO Radars.

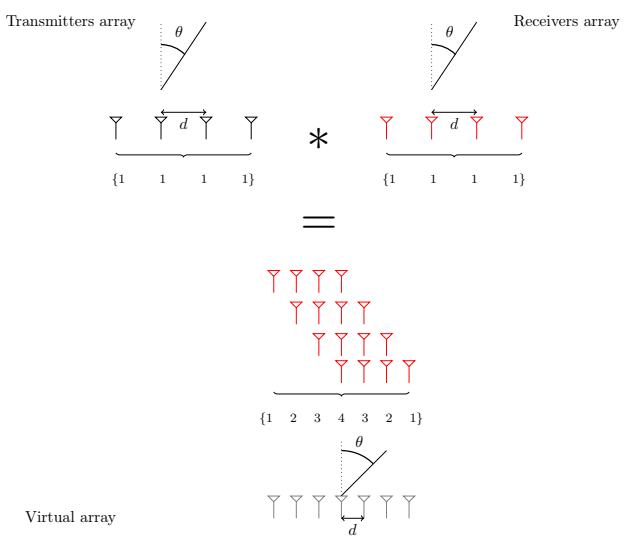

Figure 4: MIMO Virtual array.
Fig. 5 shows a beamforming comparison when Fig. 3 array is used in conventional radars and in MIMO radar.
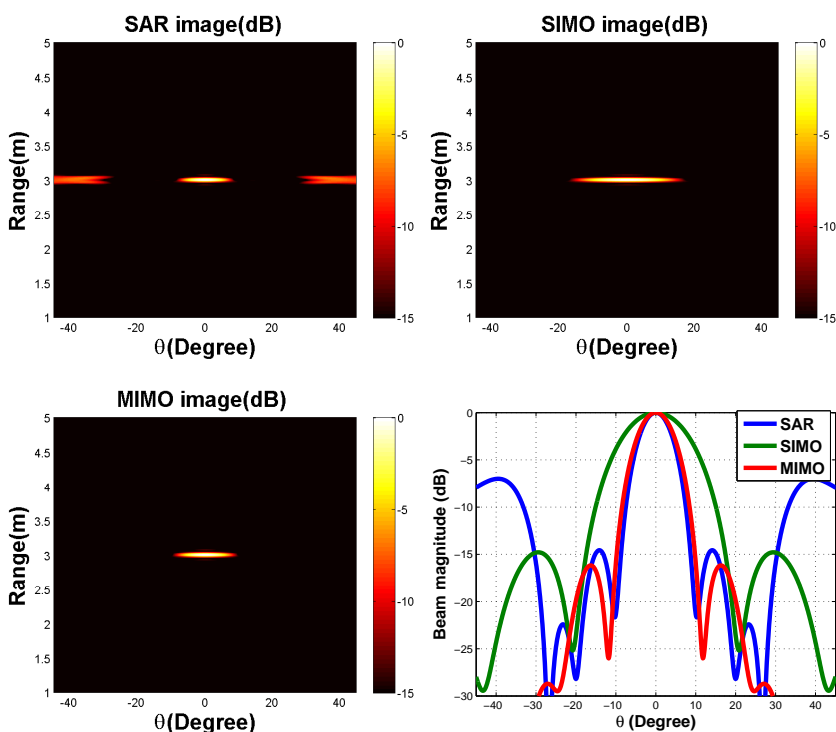

Figure 5: SAR,SIMO and MIMO imaging results.

The computing results show that the SAR provides a better angular resolution $\left(10^{\circ}\right)$ compared to the SIMO radar $\left(18^{\circ}\right)$ but the sidelobes level are very high. In contradiction, the SIMO radar presents lower sidelobes but the angular resolution is worse. Thus the MIMO radar, presents the same resolution as the SAR and the sidelobes levels a lower.

In the case of a bistatic array, the MIMO radar resolution can be improved with an appropriate array design. Fig 6 shows the beamforming results with different inter-element spacing while keeping the same number of antennas. Consequently, a sparse array can be used in MIMO radars to create a filled virtual array which provides a better angular resolution.

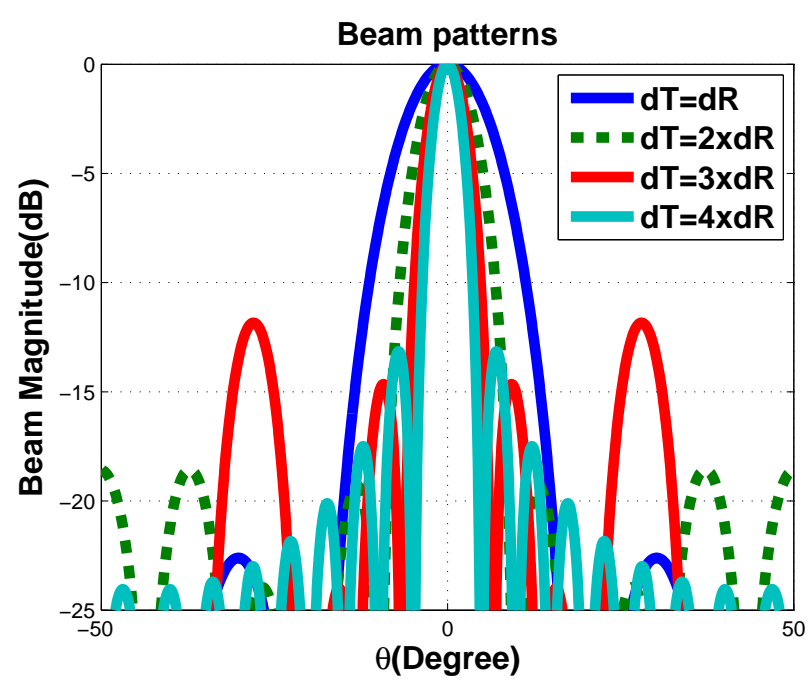

Figure 6: Angular resolution comparison with different inter-element spacing. 


\section{MEASUREMENT RESUlTS}

The presented MIMO theory has been validated using a monostatic linear array. Fig.7 and Fig.8 show the measurement setup used for the experimentation. The array consists of 4 transmitters and 4 receivers Vivaldi antennas arranged along azimuth with an inter-element spacing of $d=0.7 \times \lambda_{c}$. A foam plate is used to support the antennas. The scattering matrix is measured with a network analyzer through a $4 \times 16$ microwave switch in $3-6 G h z$ band. Two metallic cylinders with isotropic Radar Cross Sections in azimuth are placed in front of the radar system.

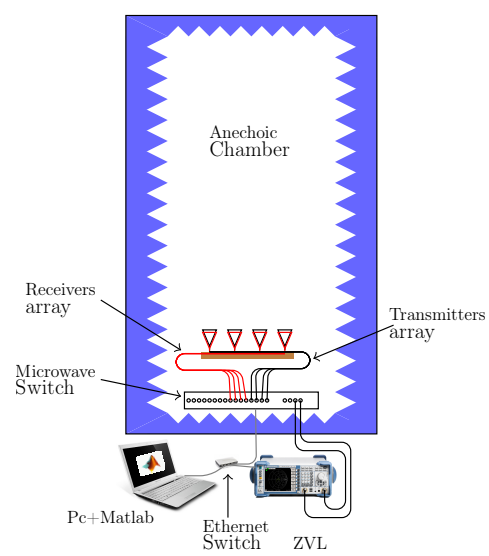

Figure 7: Experiment measurement synoptic.

In such setup the coupling signal between antennas and the delay due to cables must be calibrated in order to extract only the echo reflected by the targets. The scattering matrix used for the beamforming is then given by the equation 3 :

$$
(H)_{m, n}=\frac{\left(H_{\text {measured }}-H_{\text {coupling }}\right)}{H_{\text {cable }}}
$$

Where :

$H_{\text {cable }}$ : Scattering matrix due to the cables.

$H_{\text {coupling }}$ : Scattering matrix measured without any target.

$H_{\text {measured }}$ : Scattering matrix measured in presence of targets.

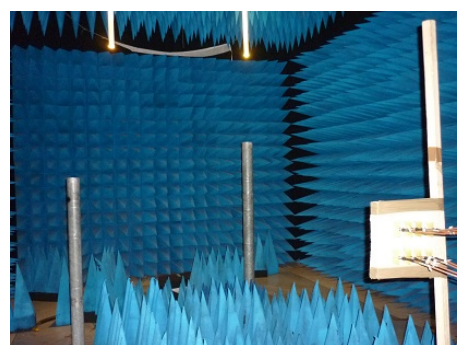

Figure 8: Measurement setup of $4 \times 4$ Transmitters/Receivers Vivaldi antennas. Two metallic cylinders are placed in the anechoic chamber in front of the transceivers array.

A digital beamforming is applied to focus the beam in the direction of the targets. MIMO imaging case supports the presented theory. It presents the best compromise resolution/sidelobes. Whereas SAR imaging present a high level of sidelobes with an accuracy angle resolution and SIMO ones presents a worse resolution with lower sidelobes level. The influence of the antennas radiation pattern is considered in the beamforming, which explains the difference between the theoretical and the experimental curves.

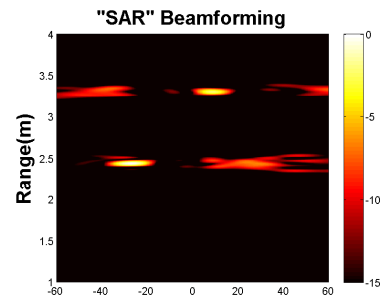

Azimuth angle(degree)
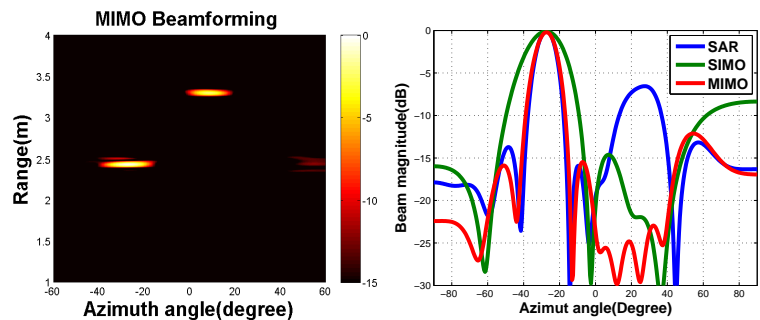

Figure 9: Imaging results of two metallic cylinders.

In addition, the resolution can be improved keeping the same number of antenna and those, thanks to the optimization of antennas array [3]. As an example, an other transceiver array has been set up with the same number of elements. In this case, the transmit array has been split in two subarrays spaced of $N \times 0.7 \times \lambda_{c}$. A same scenario is tested with two different arrays as shown in Fig.10. Imaging results show the performances of the second array. The angle resolution is improved compared to the first one so that the angle estimation of the target is more accurate. The second element spacing is chosen in order to avoid information redundancy in the scattering matrix.

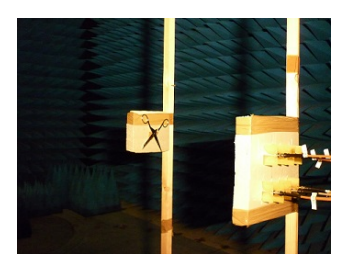

(a)

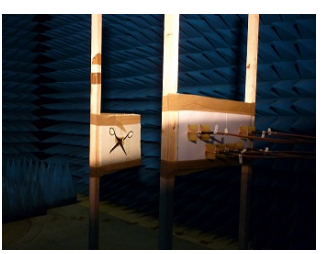

(c)

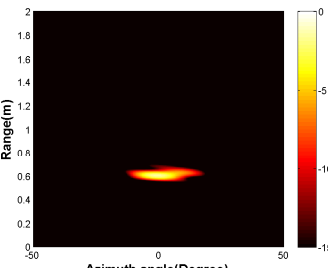

(b)

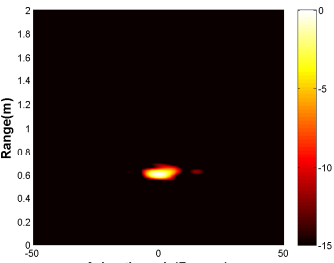

Figure 10: Imaging results of a pair of scissors from two different MIMO setups. (a) Photo of the regular spacing array. (b) Imaging result of the scissors using regular spacing array. (c) Photo of the irregular spacing array. (d) Imaging result of the scissor using the irregularly spacing array. 


\section{CONCLUSION}

In this paper, an experimental MIMO system has been presented. A first antenna array was used to highlight the benefits of MIMO radars in comparison of conventional radars. The experiment results showed that MIMO radar increases the degree of freedom and improves angle accuracy. Furthermore, MIMO radars can reach a same resolution as traditional radars with a smaller number of antennas, reducing drastically the number of elements needed. Some other measurements were set up to exhibit the discrimination capability of MIMO radar but are not presented in this paper. Future works will focus on the generation of orthogonal waveforms to probe the channel simultaneously and fast nearfield radar imaging algorithms development for high image quality.

\section{REFERENCES}

[1] P. P. Vaidyanathan and P. Pal, "MIMO radar, SIMO radar, and IFIR radar: a comparison", in Signals, Systems and Computers, 2009 Conference Record of the Forty-Third Asilomar Conference on, 2009, pp. 160-167.

[2] D. W. Bliss and K. W. Forsythe, "Multiple-input multiple-output (MIMO) radar and imaging: degrees of freedom and resolution", in Signals, Systems and Computers, 2004. Conference Record of the Thirty-Seventh Asilomar Conference on, 2003, vol. 1, pp. 54-59.

[3] Li Jian and Stoica Petre, "MIMO radar signal processing". J. Wiley \& Sons 2009.

[4] W.-Q. Wang and H. Shao, "Phased-MIMO radar with frequency diversity for increased system flexibility," in Signal Processing, Communication and Computing (ICSPCC), 2012 IEEE International Conference on, 2012, pp. 16-19.

[5] S. Ying, Z. He, H. Liu, L. Jun, and S. Gao, "Binary orthogonal code design for MIMO radar systems,” in Intelligent Signal Processing and Communication Systems (ISPACS), 2010 International Symposium on, 2010, pp. 1-4. 